\title{
A two-step approach for assessing the revitalization capability of historic buildings
}

\author{
Wojciech Terlikowski ${ }^{1, *}$ \\ ${ }^{1}$ Warsaw University of Technology, Faculty of Civil Engineering, The Institute of Building \\ Engineering, ul. Lecha Kaczyńskiego 16, 00-637 Warsaw
}

\begin{abstract}
The assessment of the revitalization capacity of historic buildings is an innovative method of interdisciplinary diagnostics, in which the condition of the building is analyzed in a context of a revitalization process. This context enforces an approach which takes into account nontechnical issues, the analysis of which gives the opportunity to draw up appropriate revitalization concepts. These issues are usually described in communal revitalization programs, taking into account social, educational and health problems of the local community. Properly carried out interdisciplinary diagnostics can show how non-technical problems can be solved by technical actions concerning a building located in a revitalized area. The two-step approach for assessing the revitalization capacity is aimed at showing the current state of the existing historic building, which has a certain potential in both technical and non-technical areas, which may directly affect the process of revitalization of the area in a crisis situation. In the first stage of the analysis, we obtain the values of partial factors - legal and formal state, historical and cultural value, structural aspects, impact on human, external environment, energy efficiency, innovation and impact on the local community factors. The difference between the acquired value of the factor and its maximum value shows a potential of the building concerning a given issue. Taking it into consideration, non-technical factors can be used to increase the building's value by planning specific revitalization activities which utilize the building's potential. The article discusses the method of effectively shaping revitalization activities by raising the partial assessment factors.
\end{abstract}

\section{Interdisciplinary building diagnostics in the revitalization process}

The revitalization process, which is according to its definition an interdisciplinary process, requires an interdisciplinary approach $[1,2]$. It should be expressed during the revitalization investment preparation period, through the diagnosis of the revitalization process, including interdisciplinary research concerning the area in a crisis situation, as well as in the entire spectrum of revitalization activities. These include engineering and technical activities related to urban planning, architecture, construction and non-technical activities such

\footnotetext{
${ }^{*}$ Corresponding author: w.terlikowski@il.pw.edu.pl
} 
as economic, environmental and social issues related to culture and education. Interdisciplinary research usually has effect in the form of revitalization programs developed at the level of whole cities or local districts. Municipal and communal revitalization programs are aimed to identify crisis areas in a city or district and take appropriate actions to both overcome negative phenomena and lead to the sustainable development of whole areas. Due to the specificity of the problem, these are long-term programs, but with a need of current updating. They are the basis for preparing Local Revitalization Programs for selected crisis areas defined in the document. Local Revitalization Programs are then prerequisites for granting financial resources from European Union structural funds.

In order to determine the crisis areas, an analysis at the scale of the whole city, district or its part are prepared, taking into account the criteria from various fields including:

1) economic criteria - for example low level of entrepreneurship, poor condition of local enterprises, lack of prepared and equipped investment areas, low level of tourist and recreational infrastructure, lack of renewable energy sources;

2) social criteria - for example high level of unemployment of inhabitants, level of affluence and poverty of inhabitants, lack of social infrastructure, difficult dwelling conditions;

3) environmental criteria - for example the level of land contamination, the presence of waste threatening the health and lives of residents, the presence of green and recreational areas;

4) criteria regarding culture, education, security - for example lack of educational facilities, level of education of residents, level of crime;

5) technical and spatial-functional criteria - for example the degree of degradation of buildings, technical condition of residential buildings, lack of technical solutions enabling effective use of buildings in terms of energy saving and environmental protection, insufficient equipment in technical and social infrastructure or its poor technical condition, lack of access to basic services or their poor quality, lack of adaptation of urban solutions, low level of communication service, lack or poor quality of public areas.

Revitalization of buildings located in an area in a crisis situation, especially residential and public buildings, which are often historic buildings, is a very important and significant element of the whole process. It results from a role of buildings in revitalized areas. Historical buildings and other construction objects located in the crisis area are usually their important part, constituting its value and revitalization potential [3]. Very often the improvement of the technical condition of these buildings is a basic technical activity necessary to be carried out, so that the revitalization process of the entire area around the building can be effective and efficient [4]. The important part of establishing a technical activities plan that addresses historical buildings is technical monitoring of structures [5]. There are numerous expert systems that can help and provide necessary solutions [6]. Therefore, the interdisciplinary nature of the revitalization process forces the interdisciplinary character of the diagnosis of revitalized buildings. An example of an interdisciplinary diagnostics is the assessment of the revitalization capability of a building, in particular a historic one. It is a diagnostics, taking into account technical issues resulting from the classic diagnostics of buildings (leading to the assessment of the technical condition) and those arising from the principles of sustainable development [7]. The principles of sustainable development in civil engineering, showing the close connection of issues related to economics, society and the environment, should obviously be present in every process of revitalization [8]. 


\section{Partial factors used in the assessment of revitalization capability of historic buildings}

The revitalization capacity of the building (including the historic one) is a set of features, properties and states of the building, in the aspects of structure, form, function, location, environmental, social and health values, that specify the possibility and the economic viability of the planned revitalization. Evaluation of the revitalization capacity of a building takes into account all aspects of revitalization including the principles of sustainable development. The value of the revitalization capacity of a historic building can be specified by the formula (1), which is given in percentage points.

$$
Z d_{\text {rew }}=\frac{\sum\left(O_{i} \cdot g_{i}\right) \cdot 100}{\sum\left(O_{i, \text { max }} \cdot g_{i}\right)}
$$

where: $\mathrm{O}_{\mathrm{i}}-$ evaluation of $\mathrm{i}$-aspect, $\mathrm{g}_{\mathrm{i}}-$ weight of $\mathrm{i}$-aspect of evaluation, $\mathrm{O}_{\mathrm{i}, \max }-$ maximum evaluation of $i$-aspect.

The revitalization capacity of the historic building $\left(\mathrm{Zd}_{\mathrm{rew}}\right)$ is the sum of the rehabilitation capacity of the building $\left(\mathrm{Zd}_{\mathrm{reh}}\right.$ - based on classical diagnostics commonly used in construction practice) and the value of sustainability rate of the revitalization process (Wzr) (2).

$$
Z d_{\text {rew }}=Z d_{r e h}+W z r
$$

In the developed algorithm of assessment of the revitalization capacity of a historic building, about 100 characteristics and states were considered. They are organized in 8 main groups of issues: formal and legal, historical and cultural value, construction, human impact, external environment, energy efficiency, innovation, and impact on the local community. This forms a framework for an interdisciplinary diagnostic [7]. In the process of determining the revitalization capacity of a building, the characteristics and states are assessed by a specific scale (for example from 0 to 5) on the basis of supporting tables. Depending on the group of issues, specific weights are applied to the results. These weights are based on an analysis of the LEED [9] and BREEAM [10] sustainable building certification systems and the author's own experiences. The result of the analysis is obtained as the point result (percentage points) followed by a final descriptive evaluation of the revitalization capacity of the building (deficient - revitalization not recommended, sufficient - revitalization difficult with problems, good - revitalization recommended, very good - revitalization with high success rate). In addition to the score, relevant conclusions from the analysis are provided.

In order to analyze all aspects of revitalization in a more detailed manner, the partial factors of particular revitalization aspects $\left(\mathrm{W}_{\mathrm{i}}\right)$ are helpful. They give the value of the aggregate assessment of a particular aspect of revitalization in a form of a percentage, assuming a maximum aggregate rating (made up of maximum partial ratings) of $100 \%$.

The following partial factors of particular aspects of the building revitalization can be distinguished:

$\mathrm{W}_{1}$ - the legal and formal state factor,

$\mathrm{W}_{2}$ - the historical and cultural value factor,

$\mathrm{W}_{3}$ - the structural aspects factor,

$\mathrm{W}_{4}$ - the impact on human factor,

$\mathrm{W}_{5}$ - the external environment factor,

$\mathrm{W}_{6}$ - the energy efficiency factor,

$\mathrm{W}_{7}$ - the possibility of innovative solutions factor,

$\mathrm{W}_{8}$ - the impact on the local community factor. 
The structural aspects factor $\mathrm{W}_{3}$ and the energy efficiency factor $\mathrm{W}_{6}$ rates are useful for analyzing the rehabilitation capacity of the building $\mathrm{Z}_{\mathrm{dreh}}$ and relate mainly to the existing technical condition of the building. However it should be noted, that the $\mathrm{W}_{6}$ factor can also show the state that can be achieved after applying revitalization actions including, for example thermo-modernization of the building. Other factors $\mathrm{W}_{1}, \mathrm{~W}_{2}, \mathrm{~W}_{4}, \mathrm{~W}_{5}, \mathrm{~W}_{7}, \mathrm{~W}_{8}$ are useful for the analysis of the value of sustainability Wzr. When assessing these factors we can consider the issues to which they relate in two ways. Evaluation of these aspects may refer to both the existing situation of the building and its potential, as a result of revitalization actions that can be carried out. A low rating of factors may determine the bad condition of an existing building, but at the same time it may indicate a high potential that can be used after applying appropriate revitalization actions.

With the $\mathrm{W}_{1}$ partial factor, formal and legal aspects of the building and real estate located in the revitalized area are assessed and analyzed (assessment weight $5 \%$ ), such as: ownership matters, possession of land and mortgage register, mortgage charges, technical documentation, and recognition as a monument and a form of monument protection. This factor determines the legal status of the building while also showing the needs that are important from the point of view of the revitalization process (eg regularization of ownership issues or the need to prepare technical documentation). The $\mathrm{W}_{2}$ factor determines the historical and cultural value of the revitalized monument (assessment weight 2.5\%). Proper promotion and presentation of these values, when they are not widely known, can have an important educational aspect and an impact on raising the economic value (tourism, culture).

The $\mathrm{W}_{4}$ factor determines the impact on people (assessment weight 5\%). Analyzing the issues related to this factor, we assess the occurrence of factors dangerous to human health and life, such as: harmful effects of mold and fungi, occurrence of dangerous materials, harmful emission of materials, noise, and vibrations. This factor both shows the existing condition of the building and indicates necessary actions to improve or completely eliminate destructive and harmful effects.

The $\mathrm{W}_{5}$ index evaluates and analyzes the building's external environment (assessment weight $12 \%$ ). The most important aspects of the assessment of the external environment include: location, communication - external transport and its adaptation and modernization possibilities, parking spaces, bicycle routes, footpaths, green areas, integration with green areas and environment, environmental impact, amount of sewage, amount of liquid and solid waste, the way of their utilization, disposal and the possibility of their reduction, harmless and environment-friendly lighting, proper water management, and recycling of materials and waste from the building. This factor is particularly sensitive to revitalization actions and its assessment can be changed through integrated revitalization actions concerning the entire area on which the revitalized building is located.

The $\mathrm{W}_{7}$ factor refers to innovation (assessment weight 6.5\%) in material, technical and technological solutions, design, innovative devices and equipment that can be used in the revitalized building as well as innovative utility solutions. This factor reveals the potential possibilities resulting from the revitalization of the building.

$\mathrm{W}_{8}$ factor concerns impacts on the local community (assessment weight $4 \%$ ) through the impact on social problems, economic stimulation, development of entrepreneurship, impact on the development of culture, and art and education. The current - real and potential, impact resulting from the possible revitalization actions compatible with the local revitalization program may be assessed. 


\section{A two-stage method of assessing the revitalization capacity of a historic building}

The assessment of the revitalization capability of the historic building $\mathrm{Zd}_{\text {rew }}$ can be determined with varying degrees of accuracy at various levels. In a very general sense, the determination of revitalization capacity can be reduced to estimating the value of many features, deriving from the technical condition of the building, from a technical and structural aspect, as well as the characteristics resulting from the principles of sustainable development. The assessment can give an estimation of the revitalization capacity, showing strengths and weaknesses of the revitalized building. When conducting advanced interdisciplinary diagnostics, depending on the level of its accuracy and professionalism, the assessment of revitalization capacity may be more credible and give more objective results, which are not only a general estimation.

Due to the possibility of two approaches to assessment of partial factors affecting the value of the sustainable revitalization of the historic building (Wzr), detailed and accurate assessment of the revitalization capacity of the historic building should be carried out in two steps.

In the first step, the values of all partial factors are assessed based on the existing, actual state of the building at the time of the study. The value of the structural factor $\mathrm{W}_{3}$ in this case determines the actual technical condition of the building before taking any rehabilitation or repair actions. Analyzing the value of this factor as well as individual aspects of the assessment that define it, it is possible to specify in detail the scope of protection, rehabilitation, renovation and conservation works necessary to perform as part of the revitalization process and resulting from the existing technical condition of the building (in architecture, construction, installation and equipment). The assessment of other factors creating the value of sustainability of the revitalization process of the historic building (Wzr) shows the real state of the analyzed aspects and at the same time potential areas in which revitalization actions are possible, which may increase the value of factors and contribute to increasing the value of sustainability of the revitalization (Wzr). The potential shown in this way should be verified, through the provisions of the Local Revitalization Program. Actions suggested in the program should be compared with potential actions resulting from the analysis of factors determining the value of sustainability Wzr in step1.

In step 2, the value of sustainability should be re-determined (through the values of the partial factors $\mathrm{W}_{1-8}$, with the exception of the structural factor $\mathrm{W}_{3}$ ). Then an increased value of the revitalization capacity of the building is received, taking into account potential revitalization actions that can be applied in a specific building, implementing the Local Revitalization Program.

As an example of a two-step approach to assessing the revitalization capacity of a historic building through changes in the value of partial assessment factors, the analysis of a residential building at Morskie Oko Street 5 in Warsaw will be presented. In the first step, the values of partial assessment factors were determined. The results present large areas of potentially possible revitalization actions (Figure 1). The value of $\mathrm{Zd}_{\text {rew }}$ was calculated as 52.5. 


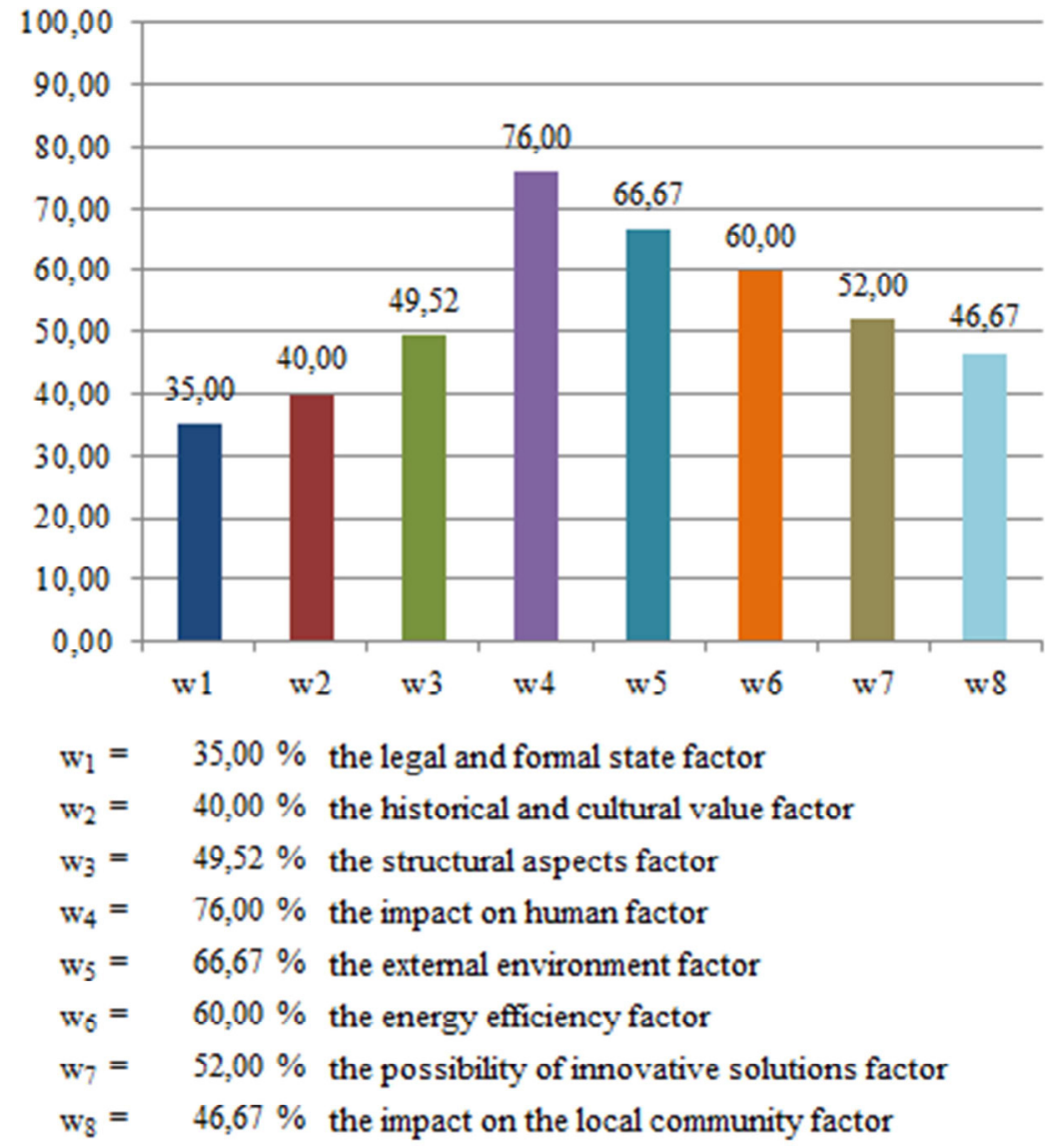

Fig. 1. Diagram of values of partial factors in Step 1 of the assessment of the $\mathrm{Zd}_{\text {rew }}$ in a residential villa at Morskie Oko Street 5 in Warsaw.

The low values of partial factors determining the value of sustainability show also the potential possibilities for their improvement through revitalization actions in the areas defined by this factors. Taking into account the recommendations of the Local Revitalization Program, it is possible to determine revitalization actions possible to implement in the areas indicated. Considering the implementation of these actions, the value of revitalization capability $\mathrm{Zdr}_{\mathrm{ew}}$ (equal 60.9) and the value of partial factors (Fig. 2), defining the value of sustainability Wzr, were redefined as a second step of analysis. 


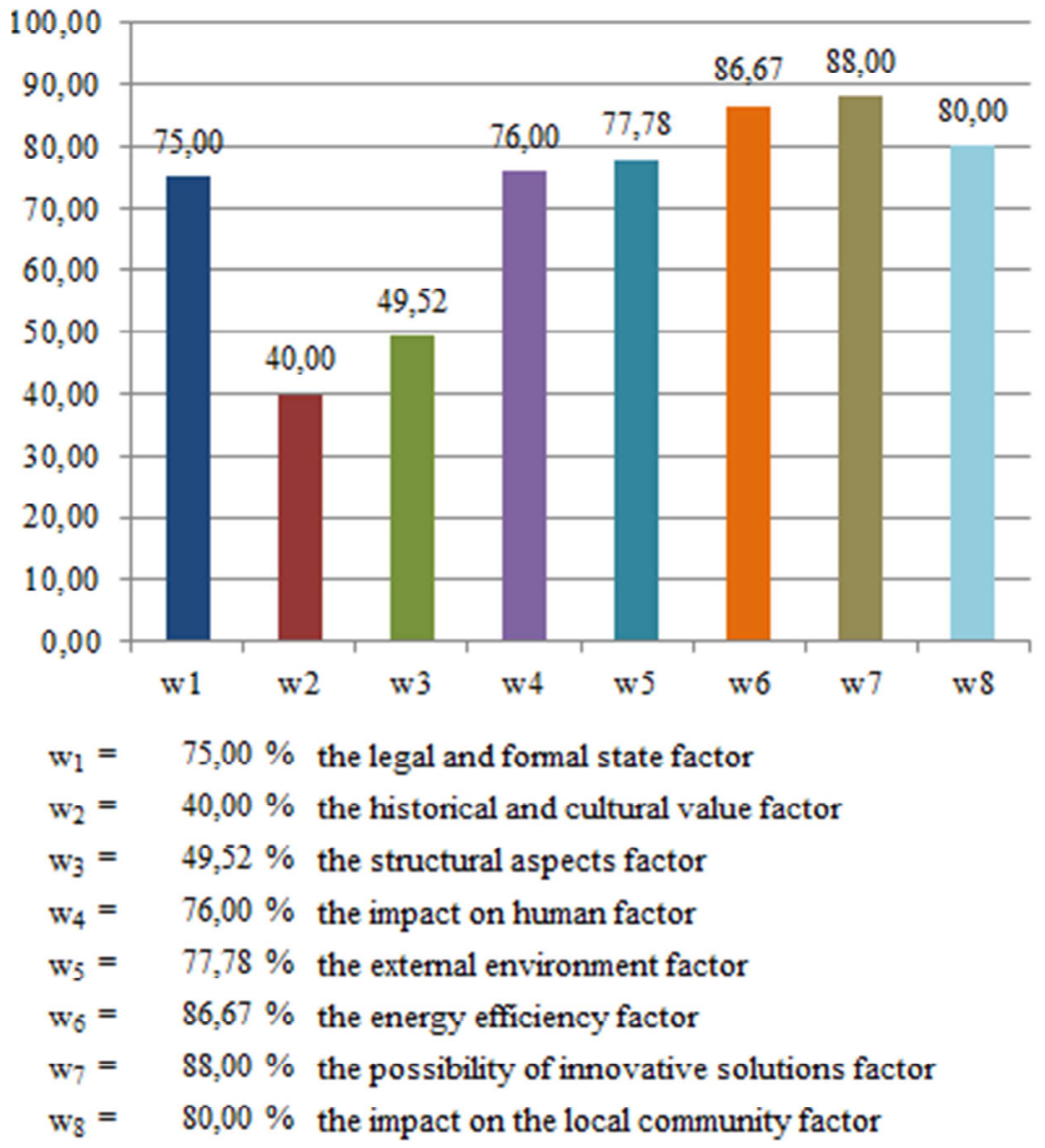

Fig. 2 Diagram of values of partial factors in Step 2 of the assessment of the $\mathrm{Zd}_{\text {rew }}$ in residential villa at Morskie Oko Street 5 in Warsaw.

\section{Summary}

Diagnostics of historic buildings should take into account the principles of sustainable development in civil engineering. This should be an interdisciplinary process, including not only the technical and structural aspects but also the non-technical ones arising from the revitalization process in which the rehabilitation of a historic building is an important element. Research conducted by the Division of Fundamentals of Buildings and Sustainable Development of Warsaw University of Technology has shown that the analysis of the revitalization potential can contribute to the ordering of knowledge about revitalized historic buildings, assessing their value in terms of planned revitalization. The two-step approach to the assessment of the building's revitalization capability, through the changes (improvement) of the values of partial factors determining the value of sustainability, gives the possibility to apply recommendations resulting from Local Revitalization Programs, by including the proposed revitalization actions. It should also be emphasized that this method, by a two-step approach and the analysis of possible changes of the value of partial factors, gives the opportunity to determine the necessary revitalization actions also when no local revitalization programs are available. This method can be useful for creating them. 


\section{References}

1. W. Terlikowski, MATEC Web Conf., 117, 00165 (2017)

2. N. Doratli, S.O. Hoskara, M. Fasli, Cities, 21, 329-348 (2004)

3. D. Dvornik Perhavec, D. Rebolj, N. Šuman, J. Cult. Herit., 16, 81-87 (2015)

4. E. Strzelecka, Bud. i Inżynieria Środowiska, 2(4), 661-668 (2011)

5. L. Runkiewicz, J. Sieczkowski, 18, 121-132 (2015)

6. A. Węglarz, P.G. Gilewski, MATEC Web Conf., 117, 00176 (2017)

7. W. Terlikowski, in 3 rd Int. Conf. Prot. Hist. Constr., (Mazzolani, F. M.) 247-248, (University of Lisbon, 2017).

8. L. Czarnecki, M. Kapron, Int. J. Soc. Mater. Eng. Resour., 17, 99-106 (2010)

9. O. Baumann, C. Reiser, J. Schafer, Bauphysik, 31, 99-105 (2004)

10. A. Ndukwe, Update, (2008) 